\title{
Vascular Endothelial Growth Factor Signaling is Required for the Behavioral Actions of Antidepressant Treatment: Pharmacological and Cellular Characterization
}

\author{
Joshua Greene ${ }^{1,2}$, Mounira Banasr ${ }^{1,2}$, Boyoung Lee ${ }^{1,2}$, Jennifer Warner-Schmidt ${ }^{1,2}$ and Ronald S Duman*,1,2 \\ 'Department of Psychiatry, Laboratory of Molecular Psychiatry, Center for Genes and Behavior, Yale University School of Medicine, New Haven, \\ CT, USA; ' ${ }^{2}$ Department of Pharmacology, Laboratory of Molecular Psychiatry, Center for Genes and Behavior, Yale University School of Medicine, \\ New Haven, CT, USA
}

\begin{abstract}
This study extends earlier work on the role of vascular endothelial growth factor (VEGF) in the actions of antidepressant treatment in two key areas. First, by determining the requirement for VEGF in the actions of a 5-HT selective reuptake inhibitor (SSRI), fluoxetine in behavioral models of depression/antidepressant response; and second, by examining the role of the 5-HTIA receptor subtype in the regulation of VEGF, and the cellular localization of antidepressant regulation of VEGF expression. The results show that pharmacological inhibition of VEGF receptor signaling blocks the behavioral actions of fluoxetine in rats subjected to chronic unpredictable stress. Infusions of SU54I6 or SUI498, two structurally dissimilar inhibitors of VEGF-Flk-I receptor signaling, block the antidepressant effects of fluoxetine on sucrose preference, immobility in the forced swim test, and latency to feed in the novelty suppressed feeding paradigm. We also show that activation of 5-HTIA receptors is sufficient to induce VEGF expression and that a 5-HTIA antagonist blocks both the increase in VEGF and behavioral effects induced by fluoxetine. Finally, double labeling studies show that chronic fluoxetine administration increases VEGF expression in both neurons and endothelial cells in the hippocampus. Taken together these studies show that VEGF is necessary for the behavioral effects of the SSRI fluoxetine, as well as norepinephrine selective reuptake inhibitor, and that these effects may be mediated by 5-HTIA receptors located on neurons and endothelial cells.

Neuropsychopharmacology (2009) 34, 2459-2468; doi:I 0.1038/npp.2009.68; published online 24 June 2009
\end{abstract}

Keywords: chronic stress; VEGF; anhedonia; antidepressant; 5-HTIA; Flk-I signaling

Basic research and clinical studies of depression and antidepressant response describe adaptive changes in several limbic brain regions, including the hippocampus (Campbell and MacQueen, 2006; Krishnan and Nestler, 2008). Much of this work has focused on adaptations of the norepinephrine and 5-HT monoamine systems, as currently available antidepressant medications block the transporters for these neurotransmitters (Krishnan and Nestler, 2008). However, increasing evidence also shows that downstream antidepressant actions include adaptations of signal transduction pathways and gene expression, notably neurotrophic factors (Martinowich et al, 2007; Schmidt and Duman, 2007). The neurotrophic hypothesis of depression is based on studies showing that stress decreases and that antidepressants increase neurotrophic factor expression in

*Correspondence: Dr RS Duman, Departments of Psychiatry and Pharmacology, Yale University, School of Medicine, Ribicoff Facilities, 34 Park St, New Haven, CT 06508, USA, Tel: + I 203974 7726, Fax: + I 203974 7724, E-mail: ronald.duman@yale.edu

Received 4 December 2008; revised 21 May 2009; accepted 22 May 2009 limbic brain structures and underlies the pathophysiology and treatment, respectively, of depression (Duman, 2004; Schmidt and Duman, 2007; Krishnan and Nestler, 2008).

Although much of this work has focused on brain derived neurotrophic factor, more recent studies also provide evidence for vascular endothelial growth factor (VEGF) in the neural mechanisms underlying the effects of stress and antidepressants (Warner-Schmidt and Duman, 2008). Stress, which can precipitate and/or exacerbate depressive episodes (Dew et al, 1987; Umberson et al, 1992), downregulates both VEGF and its major receptor in brain, Flk-1 (Heine et al, 2005). In contrast, we have reported that VEGF expression in the hippocampus is significantly and robustly increased by electroconvulsive seizure (ECS) (Newton et al, 2003). Moreover, in recent studies, we show that upregulation of VEGF is required for the neurogenic and behavioral actions of antidepressants (Segi-Nishida et al, 2008; Warner-Schmidt and Duman, 2007). We found that VEGF-Flk-1 signaling is necessary for the action of desipramine, a norepinephrine selective reuptake inhibitor (SSRI), and sufficient to induce antidepressant-like effects in behavioral paradigms used to predict antidepressant 
efficacy, including the novelty suppressed feeding and the forced swim tests (FSTs) (Warner-Schmidt and Duman, 2007). We also showed that VEGF-Flk-1 signaling is necessary for the neurogenic effects of the SSRI fluoxetine. However, the role of VEGF-Flk-1 signaling in the behavioral effects of SSRI antidepressants has not been determined. To address this issue, this study examines whether central infusions of an Flk-1 antagonist can block the behavioral effects of fluoxetine in three behavioral tests in rats exposed to chronic unpredictable stress (CUS), the sucrose preference paradigm, the FST, and novelty suppressed feeding.

We also examine the possible mechanisms underlying the induction of VEGF expression by fluoxetine, as well as the cell types that express VEGF in response to antidepressant treatment. One of several 5-HT receptor subtypes implicated in the actions of SSRIs is the 5-HT1A receptor. 5-HT1A receptor activation is required for the neurogenic and behavioral effects of fluoxetine, and administration of a 5-HT1A receptor agonist increases neurogenesis, and has behavioral effects similar to fluoxetine (Detke et al, 1995; Santarelli et al, 2003; Banasr et al, 2004). In addition, changes in 5-HT1A receptor expression, density, and/ or activity have been consistently implicated in the actions of antidepressant treatments (Blier and Ward, 2003). To examine the role of 5-HT1A receptor in the regulation of VEGF expression, we determined the effects of both an agonist (8-OH-DPAT) and a selective antagonist (WAY100635) on hippocampal VEGF protein levels. We also determined whether 5-HT1A receptor antagonist administration could block the effects of fluoxetine on VEGF and behavior. Finally, to examine the cellular source of VEGF, we conducted a double labeling study for markers of neurons and endothelial cells and VEGF mRNA expression.

\section{MATERIALS AND METHODS}

\section{Animals}

Male Sprague-Dawley rats (Charles River Labs, Wilmington, MA) weighing $225-250 \mathrm{~g}$ at the onset of experiments were paired housed in wire bottom cages under a 12-h light/dark cycle at $25^{\circ} \mathrm{C}$ with free access to food and water, except when stressors during the CUS procedure necessitated different conditions. All protocols were in strict accordance with the National Institute of Health Guide for the Care and Use of Laboratory Animals and were approved by the Yale University Animal Care and Use Committee.

\section{Antidepressants and Drugs}

For CUS/behavioral studies, rats received once daily fluoxetine $(5 \mathrm{mg} / \mathrm{kg}$, i.p., Eli Lilly, Indianapolis, Indiana) or saline, a dose shown to reverse the effect of CUS on sucrose preference and cell proliferation (Banasr et al, 2007). Fluoxetine treatment was initiated concurrently with CUS. The VEGF-Flk-1 antagonists SU5416 (4 mM, Sigma), SU1498 (4 mM, Calbiochem, San Diego, CA), or the vehicle (DMSO, Sigma) were delivered in a $1 \mu \mathrm{l}$ volume at the rate of $0.25 \mu \mathrm{l} / \mathrm{min}$ i.c.v. with a cannula (26 guage, PlasticOne, Roanoke, VA) protruding $0.5 \mathrm{~mm}$ beyond the guide cannula (PlasticOne) on days 14,16,18, and 20. The time course for
Flk-1 antagonist infusions was chosen based on earlier work showing the fluoxetine increases VEGF expression at 14 days but not at a shorter 5 day time point (Warner-Schmidt and Duman, 2007).

For VEGF levels determined by ELISA after antidepressants, there were two separate cohorts. In the first, the animals were administered sertraline $(10 \mathrm{mg} / \mathrm{kg}$, Pfizer, Groton, CT) or saline for 21 days. In the second cohort, animals were injected (i.p.) twice daily with amitriptyline $(5 \mathrm{mg} / \mathrm{kg}$, Sigma St. Louis, MO), venlafaxine $(15 \mathrm{mg} / \mathrm{kg}$, Wyeth Ayerst, Princeton, NJ), or $0.9 \%$ sterile saline for 21 days.

For 5-HT1A receptor studies of VEGF levels, 8-OH-DPAT (1 mg/kg, Sigma), WAY-100635 (1 mg/kg, Tocris, Ellisville, Missouri), or saline were injected once (i.p.) and levels of VEGF protein were determined $4 \mathrm{~h}$ later; this time point was shown to be effective for induction of hippocampal cell proliferation (Banasr et al, 2004). To determine whether the regulation of VEGF induced by 8-OH-DPAT is mediated by the 5-HT1A receptor activation, we also analyzed the effect of saline or WAY-100635 $(1 \mathrm{mg} / \mathrm{kg}) 30 \mathrm{~min}$ before the agonist.

For the CUS/WAY-100635 study, CUS rats received daily fluoxetine as described above or saline, concurrently with the start of CUS. Subgroups of animals were injected with WAY-100635 (1 mg/kg, i.p.) or saline daily starting on day 16 for 6 days. To allow for analysis of VEGF hippocampal concentrations, animals in this experiment were not subjected to FST.

For the double immuno-in situ hybridization (ISH), ECS was administered through ear-clip electrodes using a pulse generator (Ugo Basile, Comerio, Italy) (55-60 mA, 0.5-s duration, $100-\mathrm{Hz}$ frequency) to induce a generalized grand mal seizure lasting for $<15 \mathrm{~s}$. Sham animals were subjected to the same procedure but without administration of an electrical pulse. A parallel group, received either saline or fluoxetine $(5 \mathrm{mg} / \mathrm{kg})$ for 14 days, a time point at which we have reported increased levels of VEGF mRNA (WarnerSchmidt and Duman, 2007).

\section{Stereotaxic Surgeries}

Six days before the initiation of the CUS paradigm, rats were anesthetized with xylazine $(6 \mathrm{mg} / \mathrm{kg}$, i.m., Lloyd laboratories, Shenandoar, IA) and ketamine $(80 \mathrm{mg} / \mathrm{kg}$ i.m., Fort Dodge Animal Health, Overland Park, KS) and a single guide cannula was stereotaxically placed into the lateral ventricle (coordinates relative to bregma: -0.9 anteroposterior, -1.5 mediolateral, and -3.3 dorsoventral from dura (Paxinos and Watson, 1998) and held in place with aluminum screws and dental cement. Postoperative care consisted in peri-surgerical administration of carprofen $(5 \mathrm{mg} / \mathrm{kg})$ and topical triple antibiotic.

\section{Chronic Unpredictable Stress}

The protocol used for CUS was adapted from our earlier studies (Banasr et al, 2007; Warner-Schmidt and Duman, 2007) for use in animals that have undergone surgery to allow for later behavioral testing. The modifications included the exclusion of swim stress (to allow forced swim testing) and wet bedding stress (to avoid wound infection). 


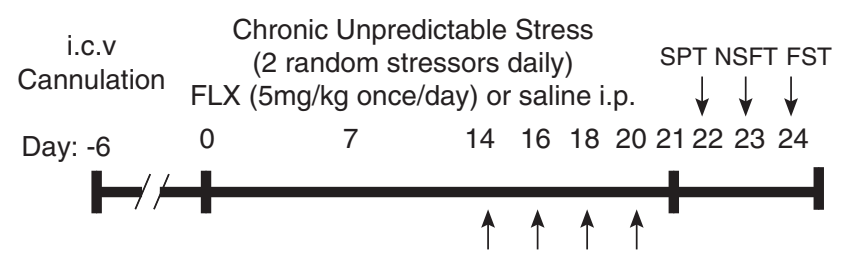

SU5416 / SU1498(1uL, 4mM) or Vehicle infused i.c.v

Figure I Protocol for CUS paradigm and drug administration. Starting 6 days after intracerebroventricular cannulation, rats were exposed to 10 stressors in a randomized sequence, twice daily for 21 days (see Materials and Methods for list of stressors). Antidepressant treatment was initiated concurrently with CUS. Rats received daily fluoxetine (FLX, $5 \mathrm{mg} / \mathrm{kg}$, i.p.) or saline. The VEGF-Flk-I antagonists, SU54I6 (4 mM), SUI498 (4 mM), or the vehicle (DMSO) were delivered in a I $\mu$ l volume at the rate of $0.25 \mu \mathrm{l} /$ min i.c.v. on days 14, 16, 18, and 20. The time course for Flk-I antagonist infusions was chosen based on earlier work showing that FLX increases VEGF expression at 14 days but not at a shorter 5 day time point (WarnerSchmidt and Duman, 2007). Starting on day 22, the ability of the Flk-I antagonists to block the behavioral effects of chronic FLX was tested in three behavioral models of antidepressant efficacy, the sucrose preference test (SPT), the novelty suppressed feeding test (NSFT), and the forced swim test (FST). The SPT, NSFT, and FST were performed sequentially so that the most stressful test was conducted last.

Animals were exposed to 10 stressors in a randomized sequence, twice daily for 21 days. The stressors used were placement in a $4^{\circ} \mathrm{C}$ environment, rotation on a shaker, lights off for $3 \mathrm{~h}$ (10 a.m.-1 p.m.), lights on overnight, strobe light overnight, aversive odor, $45^{\circ} \mathrm{C}$ tilted cages, food and water deprivation, crowding and isolation (Figure 1). The same CUS procedure was also used for the WAY-100635 study, even though there was no surgery. Control animals were handled every other day and animal weights were monitored.

\section{Sucrose Preference Test}

All behavioral testing was performed during the light cycle. Animals were habituated to a sucrose solution (1\%, Sigma) for $48 \mathrm{~h}$ (starting day 20). The sucrose preference test (SPT) was then performed after a $4 \mathrm{~h}$ water deprivation on day 22 . For $1 \mathrm{~h}$, the rats were presented with two identical bottles, one filled with the sucrose solution and the other with water. Sucrose and water consumption were determined by measuring the change in volume of fluid. Sucrose preference was calculated as percentage sucrose consumed to total fluid consumption.

\section{Novelty Suppressed Feeding Test}

After the SPT, animals were food deprived for $12 \mathrm{~h}$ and on day 23 placed in an open field $(76.5 \mathrm{~cm} \times 76.5 \mathrm{~cm} \times 40 \mathrm{~cm}$, Plexiglas) with eight pellets of food in the center. The animals were given $8 \mathrm{~min}$ to approach the food and eat. The test was stopped as soon as the animal took the first bite. The latency to eat was recorded in seconds. As a control, food consumption in the home cage was quantified.

\section{Forced Swim Test}

On day 24, rats were placed for $10 \mathrm{~min}$ in a clear cylinder with water $\left(24 \pm 1^{\circ} \mathrm{C}, 45 \mathrm{~cm}\right.$ depth), a protocol in which CUS was shown earlier to induce an increase in immobility (Haidkind et al, 2003; Banasr et al, 2008). As fluoxetine is known to reduce immobility and increase swimming (Lucki, 1997) we focused our analysis on these two behaviors. The sessions were recorded from the side and time immobile and swimming were recorded by a blind observer for the first $5 \mathrm{~min}$ of the test. Swimming was defined as movement around the tank. Immobility was when the animal made the minimal amount of movement to stay afloat. Two animals from the CUS + DMSO + SU5416 group that did not stay afloat were removed from the water and we were unable to collect data from these two animals in this test. These animals were not outliers in the other two tests and the collected data were included in the analyses.

\section{ELISA Assays}

Animals were killed by decapitation $6 \mathrm{~h}$ after AM injections on day 21 for the chronic drug study and $4 \mathrm{~h}$ after the last injection for the 5-HT1A receptor studies, brains were removed and hippocampi were dissected. Tissue samples were then quickly frozen on dry ice and stored at $-80^{\circ} \mathrm{C}$. After homogenizing by sonication, VEGF protein concentration of each sample was quantified by Elisa assay (R\&D Systems, Minneapolis, MN) using manufacturer's protocol. VEGF protein concentrations were normalized by total protein concentration as determined by BCA analysis (Pierce Chemical, Rockford, IL). Results are expressed as pg of VEGF protein/mg total protein.

\section{In Situ Hybridization}

Brains from animals subjected to chronic administration with fluoxetine (14 days) or saline and to acute ECS (Warner-Schmidt and Duman, 2007) were collected and frozen $24 \mathrm{~h}$ after the last injection of fluoxetine and 24 or $72 \mathrm{~h}$ after ECS; $14-\mu \mathrm{m}$ thick coronal sections were cut on a cryostat, thaw mounted onto slides, fixed in $4 \%$ paraformaldehyde, acetylated, and dehydrated before hybridization. VEGF riboprobe was generated by PCR using genespecific primers as described (Newton et al, 2003) and were verified by sequencing the PCR products. One microgram of the PCR product was used to make a radiolabeled riboprobe using a T7-based in vitro translation kit (Megashortscript Kit, Ambion). Sections were hybridized with the radiolabeled riboprobe $\left(2 \times 10^{6} \mathrm{cpm} / \mathrm{section}\right)$ in hybridization buffer for $18 \mathrm{~h}$ at $55^{\circ} \mathrm{C}$. Slides were washed, dried, and exposed to Kodak Biomax film (Kodak, Rochester, NY).

\section{Immunohistochemistry and ISH Double Labeling}

Studies were conducted to examine the localization of VEGF mRNA in different hippocampal cells labeled with either a neuronal (NeuN), glial (GFAP), or endothelial (RECA) cell marker. Immuno-ISH was performed as described (Newton et al, 2002). Sections were first incubated in primary antibodies against the endothelial cell marker, RECA (Serotec, Raleigh, NC) at 1:10 dilution or neuronal marker, 
NeuN (Chemicon, Temecula, CA) at 1:100 dilution, and secondary antibodies HRP conjugated antimouse (Vector Labs, Burlingame, CA). Slides were then subject to ISH as described above. Slides were dipped in emulsion solution, dried, and kept in the dark for 4 weeks before development, then counterstained with Cresyl Violet. The experiment was analyzed using Stereoinvestigator software (Microbrightfield, Williston, VT) under $\times 1000$ magnification. Background was subtracted based on the approximate number of grains counted over a comparable area in a cell-sparse region such as the hilus. Forty RECA-positive cells, 40 NeuN, or 40 GFAP-positive cells in the GCL were sampled per animal, and the number of grains was counted within each cell. Data are expressed as the percent of cells that express VEGF mRNA above background levels.

\section{Statistical Analysis}

Statistical differences were determined by analysis of the variance ANOVA (StatView 5) followed by Fisher post hoc analysis when the interaction between antidepressant $\mathrm{X}$ inhibitor was significant. The F-values and group and experimental degrees of freedom are included in the legends of the figures. For experiments with two groups, Student's $t$-test was used. The level of statistical significance was set at $P<0.05$, using two-tailed tests.

\section{RESULTS}

\section{Effects of VEGF-Flk-1 Blockade on the Antidepressant Actions of Fluoxetine}

CUS-induced anhedonia. To examine whether VEGF-Flk-1 signaling plays a role in the behavioral effects of fluoxetine, a cohort of rats was first injected with saline (SAL) or fluoxetine daily and exposed to a CUS procedure for 3 weeks, then received i.c.v. infusions of the Flk-1 antagonists or vehicle (DMSO) on days 14, 16, 18, and 20. On consecutive days starting on day 22 , the cohort was subjected to three behavioral models of antidepressant activity (Figure 1).

On day 22, all animals were subjected to the SPT, which is commonly used to assess the hedonic drive of animals after chronic mild stress (Willner, 2005). In the CUS-exposed rats we observed a level of sucrose preference $(\sim 40-50 \%$, percent sucrose to total fluid consumption) that is similar to what we have reported earlier (Banasr et al, 2007; Banasr and Duman, 2008). We found that CUS rats receiving fluoxetine showed a significant increase in sucrose preference compared with the CUS group receiving saline control $(P<0.01$, Figure $2 \mathrm{a})$, to a level $(\sim 70 \%)$ typically observed in unstressed controls. This antidepressant effect of fluoxetine was completely blocked by infusion of the VEGF-Flk-1 antagonist: the CUS + fluoxetine + SU5416 group was not significantly different from the CUS + SAL + DMSO or CUS + SAL + SU5416 groups. Two-way ANOVA statistical analysis found an interaction between drug treatment and i.c.v. infusion $(P<0.05$, Figure 2a). No significant difference between groups was observed in total fluid consumption for the $1 \mathrm{~h}$ test (CUS + SAL + DMSO: $4.5 \pm 0.7$; CUS + Fluoxetine + DMSO: $7.8 \pm 1.8 ; \quad$ CUS + SAL + SU5416: $5.2 \pm 1.3 ;$ CUS
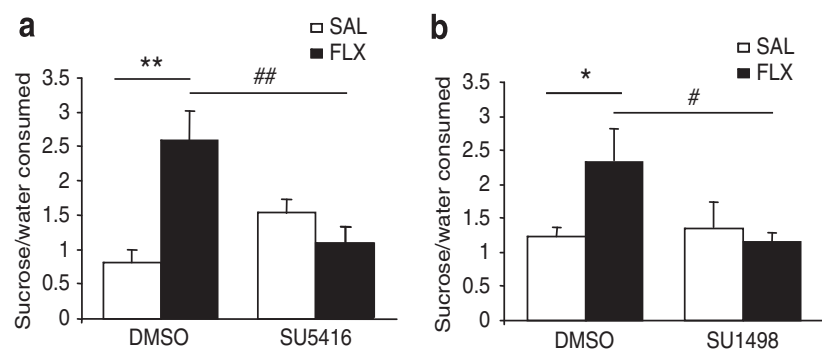

Figure 2 VEGF-Flk-I signaling is necessary for fluoxetine (FLX) reversal of CUS-induced anhedonia. (a) CUS + FLX +DMSO rats display a significantly greater preference for a sucrose solution than CUS + SAL + $\mathrm{DMSO}$ and CUS + FLX $+\mathrm{SU} 5416$ rats $(F(3,24)=5.72, P<0.01)$. There was no significant difference in sucrose consumption between CUS + FLX + SU54 16 and CUS + SAL + DMSO rats ( $n=6-9$ per group). There was a significant interaction between treatment and infusion $(F(I, 24)=12.07$, $P=0.002)$. (b) FLX had no effect on increased preference for sucrose solution in SUI498 infused CUS rats $(F(3,26)=2.59)$, but did in those infused with DMSO $(P<0.05) \quad(n=7-8$ per group). The interaction between treatment and infusion was significant $(F(I, 26)=4.72, P<0.05)$. Results are expressed as mean \pm SEM percent sucrose relative to total fluid consumption. SAL, saline control. $* P<0.05$, $* * P<0.01$ compared with CUS + SAL + DMSO; ${ }^{\#} P<0.05,{ }^{\# \#} P<0.01$ compared with CUS + $\mathrm{FLX}+\mathrm{DMSO}$.

+ fluoxetine + SU5416: $4.3 \pm 1.2$, mean \pm SEM of total fluid consumed in $\mathrm{ml}$ ).

A second cohort was exposed to the same protocol but infused with a structurally different Flk-1 antagonist, SU1498, or DMSO. In this cohort, the level of sucrose preference was higher in the CUS alone group $(\sim 50 \%)$, but fluoxetine administration resulted in a significant increase to a level typically observed in unstressed controls $(P<0.05$, Figure 2b). Infusion of SU1498, like the other VEGF-Flk-1 antagonist, completely blocked the effect of fluoxetine. Twoway ANOVA statistical analysis found an interaction between drug treatment and i.c.v. infusion (Figure $2 b$ ). There was no significant difference between groups in total fluid consumption for the $1 \mathrm{~h}$ test (CUS + SAL + DMSO: 6.1 \pm 0.4; CUS + fluoxetine + DMSO: $7.3 \pm 0.9$; CUS + SAL + SU1498: $\quad 8.6 \pm 0.8 ; \quad$ CUS + fluoxetine + SU1498: $\quad 7.4 \pm 1.1$, mean \pm SEM of total fluid consumed in $\mathrm{ml}$ ).

CUS-induced novelty suppressed feeding deficits. For the novelty suppressed feeding test (NSFT), animals were food deprived for $12 \mathrm{~h}$ after the SPT, and tested on day 23 . Latency to feed in a novel environment is responsive to chronic but not acute antidepressant treatment and is thought to measure anxiety (Santarelli et al, 2003; WarnerSchmidt and Duman, 2007).

In the first cohort, although we found that chronic fluoxetine treatment induced a $40 \%$ reduction in the latency to feed when compared with the saline treated group (Figure 3a), the two-way ANOVA statistical analysis failed to find an interaction between treatment and infusion (Figure 3a). However, in the second cohort, fluoxetine treatment led to a significant $36 \%$ decrease in latency to feed compared with saline treated CUS group (Figure $3 \mathrm{~b}$ ). In this case, the structurally different VEGF-Flk-1 antagonist completely blocked the effect of fluoxetine and there was a significant interaction between treatment and infusion $(P<0.05$, Figure $3 \mathrm{~b})$. 

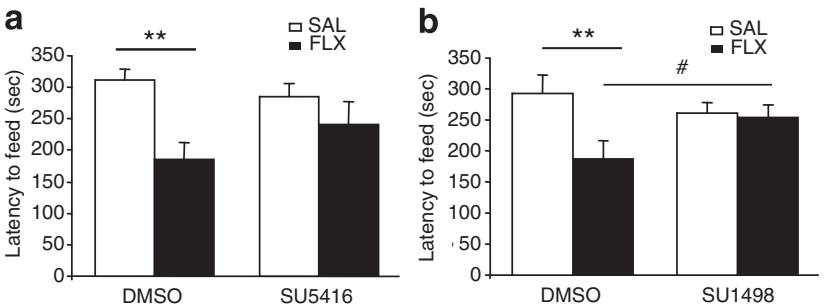

Figure 3 SUI 498 blocks the effect of fluoxetine (FLX) in the novelty suppressed feeding test. (a) Although the two-way ANOVA statistical analysis failed to find an interaction between treatment and infusion ( $F(I$, $24)=2.24, P=0.14)$, there was a significant treatment effect $(F(I$, 24) $=9.43, P=0.0052$ ) showing that CUS + FLX + DMSO treated animals shows a significantly lower latency to feed than that of the CUS + SAL + DMSO. However, the latency to feed of the CUS + FLX + SU54I6 was not significantly different from that of the CUS + SAL + SU54I6. $(n=6-9$ per group). (b) FLX had a significant effect in DMSO $(F(3,26)=3.06, P<0.0 \mathrm{I})$ but not in SUI498 treated animals $(F(3,26)=3.06, P>0 . I)(n=7-8 /$ group). The interaction between treatment and infusion was significant $(F(I, 26)=4.4 I, P<0.05)$. Results are expressed as mean $\pm S E M$ of the latency in seconds to eat. FLX, Fluoxetine; SAL, saline control. ** $P<0.0$ I compared with CUS + SAL + DMSO; ${ }^{\#} P<0.05$ compared with CUS + FLX + DMSO.

To test whether the latency changes reflected an effect on overall appetite, we measured the quantity of food consumed in the home cage during the first $10 \mathrm{~min}$ after the NSFT. There were no significant differences in levels of home cage food consumption, indicating that the changes observed in the NSFT were specific to this test (CUS + SAL + DMSO: $\quad 1.35 \pm 0.4 ; \quad$ CUS + FLX + DMSO: $0.9 \pm 0.5$; CUS + SAL + SU1498: $1.4 \pm 0.7 ;$ CUS + FLX + SU1498: $1.12 \pm$ 0.3 , mean \pm SEM in grams).

CUS-induced immobility in FST. We next examined whether VEGF-Flk-1 signaling was necessary for the effect of fluoxetine in a model traditionally used to assess antidepressant activity after an acute treatment, the FST. We found that administration of fluoxetine to CUS rats resulted in a significant decrease of $21.5 \%$ in time spent immobile $(P<0.05$, Figure $4 \mathrm{a})$ and an $81.2 \%$ increase in time spent swimming $(P<0.01$, Figure $4 \mathrm{~b})$ compared with saline treated CUS animals. Although the basal swimming was lower in the second cohort, the effect of fluoxetine was of similar magnitude in these animals (time immobile: $-20.0 \%, P<0.05$, Figure $4 \mathrm{c}$; time swimming: $+87.3 \%$, $P<0.05$, Figure $4 \mathrm{~d})$. The antidepressant effects of fluoxetine (decreased immobility and increased swimming) were blocked by administration of either SU5416 (Figure 3a and b) or SU1498 (Figure 3c and d). A $2 \times 2$ by ANOVA statistical analyses found an interaction between drug treatment and i.c.v infusion in both experiments and both analyzed parameters (immobility and swimming).

\section{Influence of Antidepressant Administration on VEGF Expression}

Influence of antidepressants. In our earlier study we found that chronic fluoxetine administration increases the expression of VEGF in the hippocampus (Warner-Schmidt and Duman, 2007). Here, we extend this work and show that chronic administration of another SSRI antidepressant, sertraline for 21 days also increases VEGF protein levels
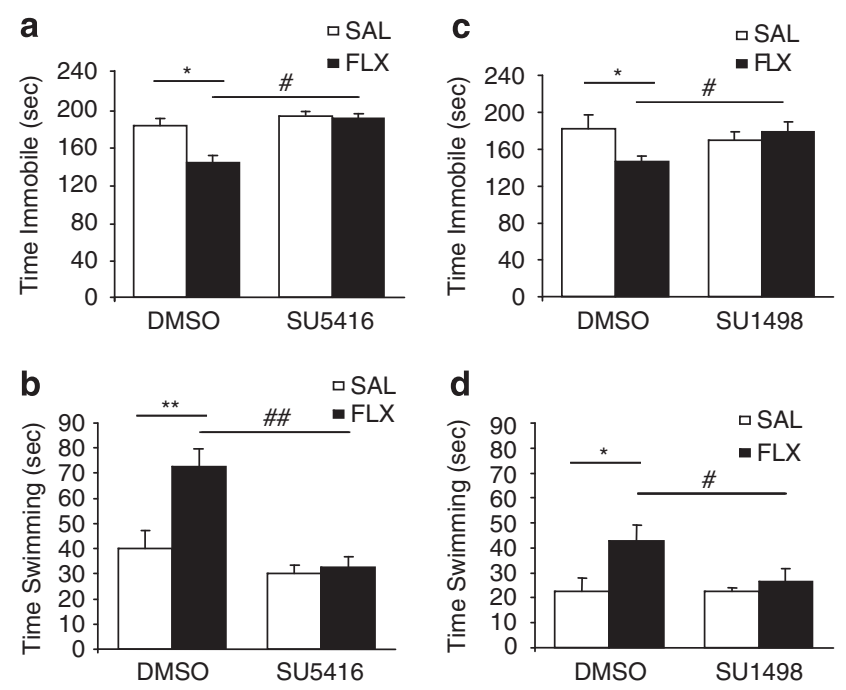

Figure 4 VEGF-Flk-I signaling is required for the effect of fluoxetine (FLX) in the forced swim test. The $2 \times 2$ ANOVA showed an interaction between the treatment $x$ infusion for both scored behaviors $(F(2,63)=$ 4.41, $P=0.016)$. (a) CUS + FLX + DMSO rats were significantly less immobile than CUS + SAL + DMSO and CUS + FLX + SU5416 rats (F(3, $2 \mathrm{I})=4.33, P<0.05)(n=4-9$ per group). Although CUS + FLX+SU54I6 and CUS + SAL + SU5416 rats did not spend significantly different times immobile. (b) CUS + FLX + DMSO swam significantly more than CUS + $S A L+D M S O(F(3,2 I)=\mid I .5 I, P<0.0 I)$, whereas there was no significant difference between CUS+FLX+SU54I6 and CUS + SAL + SU54I6. (c) CUS + FLX + DMSO rats were significantly less immobile than CUS + $\mathrm{SAL}+\mathrm{DMSO}$ and CUS +FLX+SUI498 rats $(F(3,26)=4.13, P<0.05)$ ( $n=7-8$ per group), whereas CUS + FLX + SUI 498 and CUS + SAL + SUI 498 rats did not spend significantly different times immobile. (d) CUS + $\mathrm{FLX}+\mathrm{DMSO}$ swam significantly more than CUS + SAL + DMSO (F(3, $26)=3.58, P<0.05)$, whereas there was no significant difference between $\mathrm{CUS}+\mathrm{FLX}+\mathrm{SUI} 498$ and CUS + SAL + SUI498. The $2 \times 2$ ANOVA statistical analysis found an interaction between treatment $x$ infusion for both behavioral parameters $(F(2,78)=3.7 \mathrm{I}, P=0.029)$. Results are expressed as mean \pm SEM of the seconds swimming $(a, b)$ or immobile $(c, d)$. FLX, fluoxetine; $S A L$, saline control. $* P<0.05$, **P $<0.01$ compared with CUS + $\mathrm{SAL}+\mathrm{DMSO} ;{ }^{\#} P<0.05,{ }^{\# \#} P<0.01$ compared with CUS + FLX+DMSO.

determined by ELISA of hippocampal homogenates (saline: $6.37 \pm 0.5, n=6$; sertaline: $8.13 \pm 0.23, n=5 ; t<0.01$ ). We also examined the influence of two other antidepressant drugs on levels of VEGF in the hippocampus, including a tricyclic (amitriptyline) and a dual reuptake inhibitor (venlafaxine). The results show that chronic administration of amitriptyline $(P<0.05)$ or venlafaxine $(P<0.01)$ also increases levels of VEGF protein in the hippocampus $\left(\mathrm{F}_{2,15}=5.32, P=0.017 ; n=6 /\right.$ group $)$. This work complements our earlier studies in showing that different classes of chemical antidepressants and ECS increase VEGF expression in the hippocampus (Warner-Schmidt and Duman, 2007).

Influence of 5-HT1A receptor agents. We have also initiated studies to examine the role of the 5-HT1A receptor subtype in the regulation of VEGF protein levels. Here, we show that acute administration $(4 \mathrm{~h})$ of the agonist, 8-OH-DPAT, increases levels of VEGF in hippocampal homogenates by $23 \%(t<0.05 ; n=5-6 /$ group; Table 1$)$. In contrast, acute administration $(4 \mathrm{~h})$ of the selective antagonist WAY-100635 resulted in a $16 \%$ decrease in hippocampal VEGF levels $(t<0.05) ; n=5$-6/group; Table 1$)$. 
Table I Regulation of VEGF in the Hippocampus by 5-HTIA Receptor Selective Agents

\begin{tabular}{lc}
\hline Treatment & VEGF protein levels pg/mg \\
\hline Experiment I & $10.3 \pm 0.3$ \\
Saline & $12.2 \pm 0.7^{*}$ \\
8-OH-DPAT & \\
Experiment 2 & $10.2 \pm 0.6$ \\
Saline & $8.6 \pm 0.4^{*}$ \\
WAYI00635 & \\
& \\
Experiment 3 & $12.9 \pm 0.2$ \\
Saline + saline & $15.0 \pm 0.8^{*}$ \\
Saline + 8-OH-DPAT & $12.8 \pm 0.5^{\#}$ \\
WAYI00635+8-OH-DPAT & \\
\hline
\end{tabular}

8-OH-DPAT and WAYI00635 were administrated as described in 'Materials and Methods' and levels of hippocampal VEGF were determined by ELISA. Results are expressed as mean \pm SEM pg VEGF/mg total protein. $* P<0.05$ compared with saline treated animals and ${ }^{\#} P<0.05$ compared to 8-OH-DPAT treated animals (ANOVA and Fisher's post hoc test).

In addition, we also found that pretreatment with WAY10063530 min before administration 8-OH-DPAT prevents the increase in hippocampal VEGF levels (ANOVA: $\mathrm{F}_{2,13}=4.5, P=0.032, n=5$-6/group). VEGF protein levels in the WAY-100635+8-OH-DPAT treated animals were significantly different from 8-OH-DPAT treated animals $(P<0.05)$ and not different from the control animal group (Table 1).

\section{WAY-100635 Blocks the Behavioral Actions of Fluoxetine}

To further examine the role of 5-HT1A receptor signaling in the behavioral and VEGF effects of fluoxetine, we determined if WAY-100635 could block the effects of fluoxetine in the CUS paradigm. We confirmed that CUS exposure results in a significant decrease in sucrose preference to a level $(40 \%)$ observed in our earlier work as well as this study $(P<0.01$, Figure $5 a$, compare with Figure 2$)$. In the NSFT we also observed a $77 \%$ greater latency to feed in the CUS group compared with controls $(P<0.01$, Figure $5 b)$. CUS + fluoxetine animals showed a significantly greater sucrose preference and lower latency to feed than CUS animals, effects were almost identical to control animals in both tests $(P<0.01$, Figure $5 \mathrm{a}$ and $\mathrm{b})$. These effects of fluoxetine were completely blocked by WAY-100635 as there was no significant difference in behavior between the CUS + fluoxetine + WAY-100635 and the CUS animals. No treatment group showed altered total fluid or home cage food consumptions (data not shown), as described for Figures 2 and 3.

Analysis of hippocampal VEGF levels showed that there was no effect of CUS, but that administration of fluoxetine significantly increased VEGF by $\sim 25 \%$ in CUS + fluoxetine animals compared with the CUS or control animals $\left(\mathrm{F}_{3,20}=4.11 ; P=0.02\right.$, Table 2$)$. This effect was blocked by WAY-100635, as there was no significant difference between a

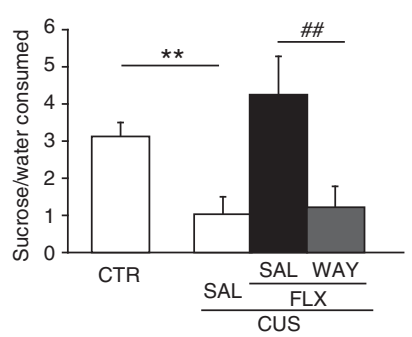

b

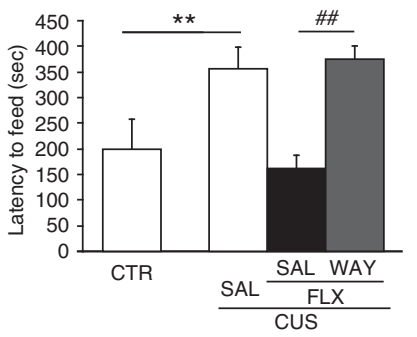

Figure 5 Behavioral actions of fluoxetine are blocked by WAY-I00635. (a) CUS rats showed a significantly lower percent of sucrose consumed than control (CTR) rats. $(F(3,20)=7.09, P<0.0 I)$ ( $n=6$ per group). CUS $+F L X$ rats show a significantly greater sucrose preference than CUS and CUS + FLX +WAY-100635 rats $(P<0.01$ and $P<0.05$, respectively). There was no significant difference between the sucrose preference of the CUS and CUS + FLX + WAY-100635 rats. (b) The latency for CUS rats to feed in a novel environment was significantly greater than the latency for CTR and CUS + FLX rats $(F(3,20)=4.33, P<0.0 I)(n=6$ per group), whereas there was no significant difference between CUS rats and CUS + FLX + WAY rats. Results are expressed as mean \pm SEM percent sucrose to total fluid consumption (a) or mean \pm SEM latency to feed in seconds (b). CTR, control; FLX, fluoxetine; WAY, WAY 100635. *** $<0.01$ compared with CTR; ${ }^{\#} P<0.01$ compared with CUS + FLX + WAY.

the CUS + fluoxetine + WAY-100635 and the CUS animals. There was also a significant difference between CUS + fluoxetine + WAY-100635 and CUS + fluoxetine $(P<0.05$, Table 2).

\section{Neuronal and Endothelial Cell Expression of VEGF is Increased by Fluoxetine}

Our earlier ISH studies show that VEGF mRNA expression is increased in the granule cell layer of the dentate gyrus by chronic fluoxetine administration or by ECS (Newton et al, 2003; Warner-Schmidt and Duman, 2007). To determine what cell types in the GCL express VEGF mRNA in response to antidepressant treatment, a double immunohistochemistry/ISH procedure was performed to assess the colocalization of VEGF mRNA with NeuN-positive neurons (Figure 6a and b), RECA-positive endothelial cells (Figure $6 \mathrm{c}$ and d), or GFAP-positive cells (Figure 6e and f). As ECS produces a robust induction of VEGF mRNA, as well as neurogenesis, we also compared the effects of this treatment on the cellular localization of VEGF expression. For this experiment, fluoxetine was administrated for 14 days, which corresponds to the time at which we have observed an increase in levels of VEGF mRNA (Warner-Schmidt and Duman, 2007) and started inhibitor infusions. As predicted based on our earlier studies, the percent of NeuN-positive cells expressing VEGF was significantly increased by chronic fluoxetine treatment compared with saline controls (Figure 6g). In addition, there was a significant increase in VEGF mRNA in RECA-positive endothelial cells after fluoxetine administration (Figure 6g).

A single ECS significantly increased the number of NeuNpositive cells expressing VEGF mRNA in the GCL compared with sham-handled controls $24 \mathrm{~h}$ later, but by $72 \mathrm{~h}$ levels were the same as sham (Figure 6a, b and $h$ ). There was also a significant four-fold increase in the number of RECApositive endothelial cells that express VEGF mRNA at the 
Table 2 Effect of WAY 00635 on Hippocampal VEGF Protein Levels in Animals Subjected to CUS and Fluoxetine Treatment

\begin{tabular}{lc}
\hline Treatment & $\begin{array}{c}\text { VEGF protein } \\
\text { levels pg/mg }\end{array}$ \\
\hline CTR & $12.6 \pm 0.7$ \\
CUS & $12.0 \pm 0.5$ \\
CUS +FLX & $15.4 \pm 0.9 * *$ \\
CUS +FLX+WAYI00635 & $13.2 \pm 0.6^{\#}$ \\
\hline
\end{tabular}

Fluoxetine and WAYI 00635 were administrated as described in the 'Materials and Methods' and levels of hippocampal VEGF were determined by ELISA.

Results are expressed as mean \pm SEM pg VEGF/mg total protein. CTR, control; CUS, chronic unpredictable stress; FLX, fluoxetine. $n=6$ per group. $* 0.0$ I compared with CUS, ${ }^{\#}<0.05$ compared with CUS + FLX (ANOVA and Fisher's post hoc test).

$24 \mathrm{~h}$ time point (Figure $6 \mathrm{c}, \mathrm{d}$ and $\mathrm{h}$ ). Interestingly, after $72 \mathrm{~h}$, the amount of endothelial-expressed VEGF mRNA was still elevated almost three-fold, although this effect was not significant $(P=0.06$, Figure 6f). VEGF is also expressed in GFAP-positive astrocytes, but there was no significant induction of VEGF in this population of cells with either fluoxetine or ECS treatment (Figure 6e, $\mathrm{f}$ and $\mathrm{h}$ ).

\section{DISCUSSION}

This study shows that VEGF-Flk-1 signaling is required for the behavioral effects of the SSRI antidepressant fluoxetine in three different behavioral paradigms. We show that blockade of Flk-1 signaling alters the effects of chronic fluoxetine on CUS-induced deficits in the SPT and FST, and attenuate the effects of the SSRI on the NSFT. In addition, the results show that SSRIs increase VEGF expression in the hippocampus and that fluoxetine induction of VEGF occurs in both neurons and endothelial cells. We also found that acute administration of the 5-HT1A receptor agonist 8-OHDPAT increases VEGF protein levels, whereas treatment with the antagonist, WAY-100635, reduces VEGF levels and blocks the effects of fluoxetine on sucrose preference and novelty suppressed feeding.

FST and NSFT are typically used in nonstressed animals to predict the efficacy of drugs as antidepressants and measure helplessness- and anxiety-like behaviors, respectively (Bodnoff et al, 1989; Lucki, 1997). We have shown earlier that animals subjected to CUS show increased latency to feed in NSFT and immobility in the FST (Haidkind et al, 2003; Banasr et al, 2008), as well as deficits in SPT, an index of anhedonia (Papp et al, 1991; Muscat et al, 1992; D'Aquila et al, 1997). Here, we confirm that chronic fluoxetine administration increases sucrose preference in rats exposed to CUS, and also decreases latency to feed and immobility. This validates the CUS paradigm as a model in which stress-induced behaviors are counteracted by chronic treatment with fluoxetine.

The effects of VEGF in brain are mediated by two highaffinity tyrosine kinase receptors for VEGF, Flk-1, and Flt-1 (de Vries et al, 1992; Quinn et al, 1993). We focused on Flk-1 because this receptor subtype is expressed by neural progenitor and endothelial cells throughout the hippocampus
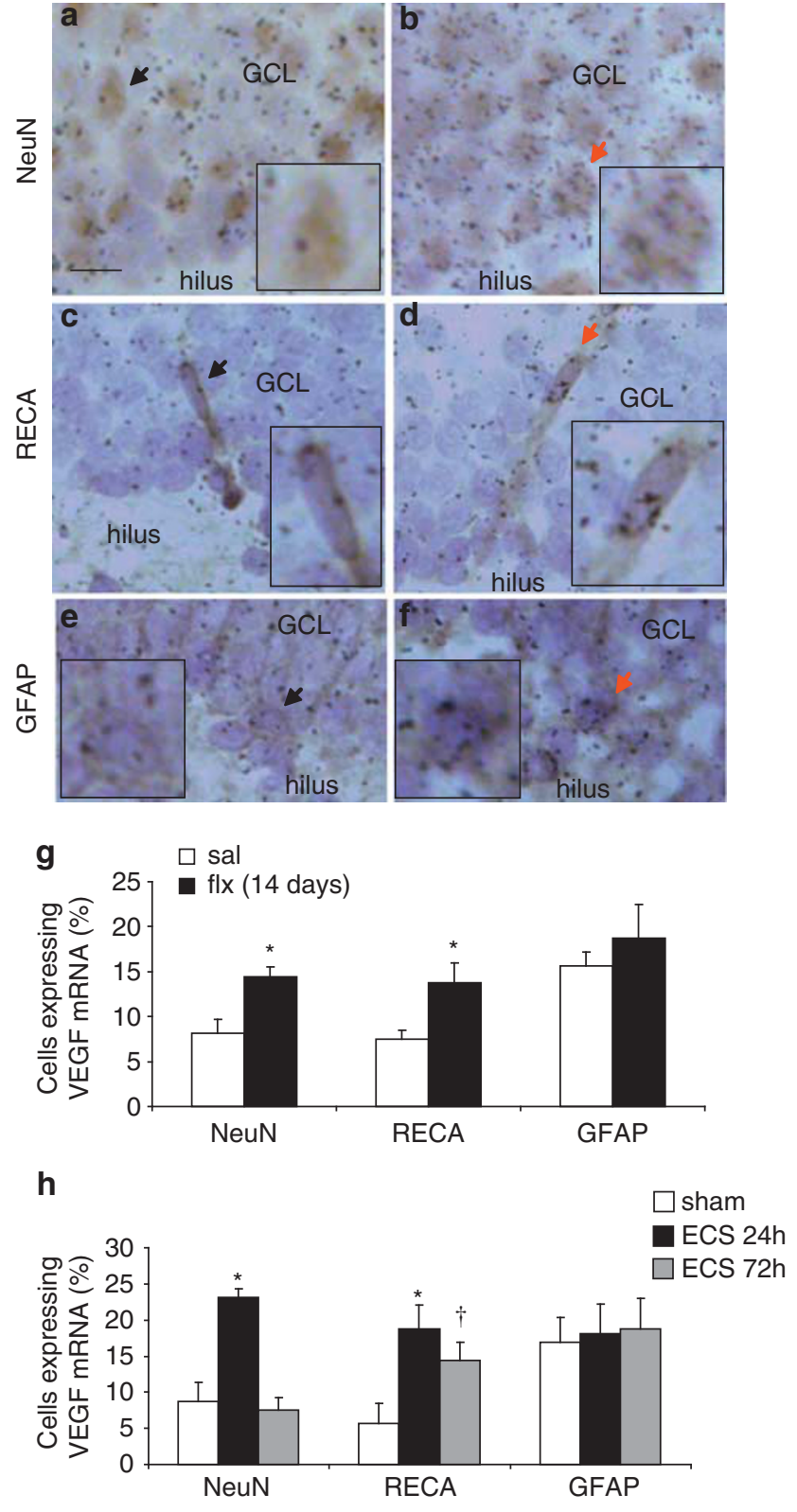

Figure 6 VEGF mRNA is expressed by neurons and endothelial cells: regulation by ECS and fluoxetine treatment. Immunohistochemistry with phenotypic markers of neurons (NeuN) or endoethelial cells (RECA) followed by in situ hybridization for VEGF mRNA (black grains). (a, b) Representative images of the GCL cells immunolabeled with either NeuN $(a, b)$, RECA (c, d) (brown) or GFAP antibody (e, f) counterstained with cresyl violet (blue); Scalebar $=10 \mu \mathrm{m}$. Sections are from control (a, c, e) or ECS (b, d, f). In all images, arrows indicate NeuN-, RECA-, or GFAPpositive cells expressing VEGF mRNA. Background was defined as 3 grains per cell, which was the approximate number of grains counted over a comparable area in a cell-sparse region such as the hilus. Inset images highlight the arrow-marked cell at 200\% magnification. (g) Quantification of the influence of fluoxetine (FLX) on the number of cells expressing VEGF mRNA above background levels the in the $G C L\left(N e u N: F_{(1,6)}=10.000\right.$, $P<0.05$; RECA: $\left.F_{(1,6)}=6.818, P<0.05\right)$. (h) Quantification of the influence of ECS on the number of cells expressing VEGF mRNA in the GCL (NeuN: $\left.F_{(2,9)}=19.966 ; \quad P<0.01 ; \quad R E C A: \quad F_{(2,9)}=5.304 ; \quad P<0.05\right)$. $* P<0.05$, $\dot{\dagger} P=0.06$ compared with saline or sham ECS.

(Yang et al, 2003) and because Flk-1 signaling is necessary for the neurogenic effects of fluoxetine (Warner-Schmidt and Duman, 2007). We found that two structurally different Flk-1 
antagonists blocked the behavioral effects of fluoxetine in CUS animals in the SPT and the FST. The effects of fluoxetine administration in the NSFT were also blocked by inhibition of Flk-1 signaling, but a significant drug $\mathrm{x}$ antagonist interaction was only observed for SU1498. The demonstration that SU1498 blocks the behavioral actions of fluoxetine also extends our earlier work on SU5416 (Warner-Schmidt and Duman, 2007). The latter compound is relatively selective for Flk-1, but also inhibits other receptor tyrosine kinases, including stem cell factor receptor c-Kit (Smolich et al, 2001) and Ret tyrosine kinase (Mologni et al, 2006), albeit with lower affinity than Flk-1. In contrast, SU1498 has little or no effect on c-Kit and Ret, providing strong evidence that VEGF-Flk-1 signaling is necessary for the behavioral actions of fluoxetine.

The hippocampus has been one of the key regions implicated in depression and antidepressant treatment. Brain imaging studies reveal that the volume of this limbic brain structure is significantly decreased in patients with major depressive disorder (MacQueen et al, 2003) and that hippocampal volume decreases are inversely related to the length of antidepressant treatment (Sheline et al, 2003). It has been hypothesized that the downregulation of neurotrophic factors could contribute to the volume reduction (Duman, 2002) and conversely that the upregulation or restoration of neurotrophic factor expression could mediate some aspects of antidepressant action (Duman, 2004). This neurotrophic hypothesis of depression and antidepressant action, based largely on BDNF, has recently been extended to other growth factors, most notably VEGF (WarnerSchmidt and Duman, 2008). We have reported that fluoxetine, despramine, and ECS all increase VEGF expression in the hippocampus. Here, we show that several other antidepressants including another SSRI (sertraline), a dual reuptake inhibitor (venlafaxine), and a tricyclic (amitriptyline), at doses known to be effective on classical behavioral tests of antidepressant efficacy (Rogoz et al, 2002; Weinstock et al, 2002; West and Weiss, 2005; Kulkarni et al, 2008; Sell et al, 2008) also increase VEGF protein levels in the hippocampus. Taken together with our earlier report, these data indicate that VEGF is a common target of chemical and nonchemical antidepressants. However, given the route of administration of the Flk-1 blockers (i.c.v.), it is possible that VEGF signaling in other brain regions also contributes to the actions of fluoxetine and the effects of the inhibitors.

This study also provides evidence that activation of 5-HT1A receptors underlies the actions of SSRI antidepressants. We found that acute administration of a nonselective 5-HT1A receptor agonist 8-OH-DPAT increases VEGF expression $4 \mathrm{~h}$ after administration, a time point that also increases neurogenesis and produces antidepressant-like effects in the FST (Detke et al, 1995; Banasr et al, 2004). Conversely, acute administration of a highly selective 5-HT1A receptor antagonist, WAY-100635, decreases VEGF protein levels, suggesting that there is a basal level of 5-HT1A activation that contributes to VEGF expression. In addition, the ability of WAY-100635 to block the effect of 8-OH-DPAT shows that the effects of this nonselective agonist are mediated through the 5-HT1A receptor. The mechanism underlying 5-HT1A receptor-stimulation of the VEGF expression is unclear. The VEGF gene contains a cAMP response element (CRE) and expression can be stimulated by activation of the CRE-binding protein (CREB; Impey et al, 2004). Although 5-HT1A inhibition of the cAMP cascade (De Vivo and Maayani, 1990) is inconsistent with a role for this pathway, 5-HT1A receptors are also reported to stimulate other pathways (eg, ERK and Akt) that can lead to activation of CREB and VEGF expression (Mendez et al, 1999; Cowen, 2007). Additional studies will be required to determine the role of these pathways in the regulation VEGF expression by 5 -HT1A receptors and SSRI antidepressants.

In addition to this study a role for 5-HT1A receptors in the action of antidepressant treatment is supported by several lines of evidence. Recent studies show that 5-HT1A receptor activation is required for the effects of antidepressants, including fluoxetine, on neurogenesis and on neurogenic-dependent behaviors (Santarelli et al, 2003; Holick et al, 2008). However, one of these studies shows that the role of 5-HT1A receptors is strain specific in mice, raising a question regarding a definitive role of 5-HT1A receptors in the neurogenic and behavioral actions of antidepressants (Holick et al, 2008). Upregulation of 5-HT1A receptor density and/or activity after chronic antidepressant treatment has been suggested to contribute to the delayed therapeutic effect of antidepressants (Blier, 2001; Blier and Ward, 2003). In addition, a role for 5-HT1A receptors in the behavioral and neurogenic effects of fluoxetine has been reported. These effects are strain specific (Holick et al, 2008). Together, the rapid induction of VEGF expression in response to 8-OH-DPAT, suggests a mechanism through which antidepressant-induced 5-HT1A receptor activation can increase neurogenesis and alter depressive-like behavior in rat.

In the CUS paradigm, we found that subchronic treatment with WAY-100635 blocks the effect of fluoxetine on SPT and NSFT. The timing of WAY-100635 administration was chosen based on the time course for fluoxetine regulation of 5-HT1A receptor activity (Blier, 2001) and VEGF expression (Warner-Schmidt and Duman, 2007). We also found that fluoxetine administration increased levels of VEGF in CUS animals, and that this effect was blocked by administration of WAY-100635. This is consistent with the behavioral findings and the hypothesis that induction of VEGF underlies the actions of antidepressants. However, CUS alone did not significantly decrease levels of VEGF, as reported earlier (Heine et al, 2005), indicating that the CUSinduced behavioral deficits do not result from reduced VEGF protein. Taken together the results suggest that 5HT1A receptor induction of VEGF is required for the behavioral responses to fluoxetine, but that decreased VEGF is not required for CUS-induced behavioral responses.

The co-localization studies show that chronic fluoxetine administration significantly increases VEGF expression in neuronal and endothelial cells. These effects were compared with ECS, which stimulates the largest induction of VEGF expression and neurogenesis of antidepressants tested (Warner-Schmidt and Duman, 2007, Segi-Nishida et al, 2008). ECS also increases neuronal and endothelial cell expression of VEGF, albeit to higher levels than fluoxetine. In addition, the induction of VEGF in endothelial cells, but not neurons is sustained (ie at $72 \mathrm{~h}$ after ECS) and correlates with the time course for increased cell proliferation (Warner-Schmidt and Duman, 2007). The induction of 
VEGF in endothelial cells is particularly interesting given the evidence for neurogenesis occurring in a vascular niche (Palmer et al, 2000). Dividing cells in the adult hippocampus are often found in clusters that are associated with the vasculature, and many of these newborn cells express endothelial cell markers. These findings suggest that neurogenesis is regulated by interactions with endothelial cells and/or factors released from these cells, including VEGF (Jin et al, 2002; Cao et al, 2004). The induction of endothelial VEGF could contribute to the induction of neurogenesis by fluoxetine and ECS (Warner-Schmidt and Duman, 2007). Stimulation of neuronal VEGF could also contribute to neurogenic responses, as well as neuroprotective- (Storkebaum et al, 2004) and neuroplasticity-related effects (Cao et al, 2004). Moreover, both endothelial and neuronal VEGF could contribute to the behavioral responses observed in this study. The induction of VEGF in endothelial cells and neurons could result from activation of 5-HT receptor subtypes on these cells, including neuronal 5-HT1A receptors (Riad et al, 2000).

In summary, this study shows that VEGF-Flk-1 signaling is necessary for the behavioral actions of fluoxetine, and provides additional evidence that VEGF regulation is a common and necessary target of antidepressant treatment. In addition, the results suggest that $5-\mathrm{HT} 1 \mathrm{~A}$ activation may be involved in the antidepressant-induced regulation of VEGF expression in neuronal and endothelial cells. Studies are currently underway to characterize the localization and mechanisms by which 5-HT1A and other 5-HT receptor subtypes regulate the expression and function of VEGF in response to antidepressants.

\section{ACKNOWLEDGEMENTS}

This work was supported by United States Public Health Service Grants MH45481 and 2 PO1 MH25642, Veterans Administration National Center Grant for Post-Traumatic Stress Disorder, and the Connecticut Mental Health Center.

\section{DISCLOSURE/CONFLICT OF INTEREST}

The authors declare that except for income received from the primary employer no financial support or compensation has been received from any individual or corporate entity over the past 3 years for research or professional service aside from those listed below and there are no personal financial holdings that could be perceived as constituting a potential conflict of interests. Dr Duman has served as a consultant for Taisho, Takeda, and Psychogenics, received research grants from Organon and Sepracor, and honoraria from Lundbeck, Lilly, Bristol Myers Squibb, and Pfizer.

\section{REFERENCES}

Banasr M, Chowdhury GM, Terwilliger R, Newton SS, Duman RS, Behar KL et al (2008). Glial pathology in an animal model of depression: reversal of stress-induced cellular, metabolic and behavioral deficits by the glutamate-modulating drug riluzole. Mol Psychiatry [e-pub ahead of print].

Banasr M, Duman RS (2008). Glial loss in the prefrontal cortex is sufficient to induce depressive-like behaviors. Biol Psychiatry 64: 863-870.
Banasr M, Hery M, Printemps R, Daszuta A (2004). Serotonininduced increases in adult cell proliferation and neurogenesis are mediated through different and common 5-HT receptor subtypes in the dentate gyrus and the subventricular zone. Neuropsychopharmacology 29: 450-460.

Banasr M, Valentine GW, Li XY, Gourley SL, Taylor JR, Duman RS (2007). Chronic unpredictable stress decreases cell proliferation in the cerebral cortex of the adult rat. Biol Psychiatry 62: 496-504.

Blier P (2001). Pharmacology of rapid-onset antidepressant treatment strategies. J Clin Psychiatry 62(Suppl 15): 12-17.

Blier P, Ward NM (2003). Is there a role for 5-HT1A agonists in the treatment of depression? Biol Psychiatry 53: 193-203.

Bodnoff SR, Suranyi-Cadotte B, Quirion R, Meaney MJ (1989). A comparison of the effects of diazepam versus several typical and atypical anti-depressant drugs in an animal model of anxiety. Psychopharmacology (Berl) 97: 277-279.

Campbell S, MacQueen G (2006). An update on regional brain volume differences associated with mood disorders. Curr Opin Psychiatry 19: 25-33.

Cao L, Jiao X, Zuzga DS, Liu Y, Fong DM, Young D et al (2004). VEGF links hippocampal activity with neurogenesis, learning and memory. Nat Genet 36: 827-835.

Cowen DS (2007). Serotonin and neuronal growth factors - a convergence of signaling pathways. J Neurochem 101: 1161-1171.

D'Aquila PS, Newton J, Willner P (1997). Diurnal variation in the effect of chronic mild stress on sucrose intake and preference. Physiol Behav 62: 421-426.

De Vivo M, Maayani S (1990). Stimulation and inhibition of adenylyl cyclase by distinct 5-hydroxytryptamine receptors. Biochem Pharmacol 40: 1551-1558.

de Vries C, Escobedo JA, Ueno H, Houck K, Ferrara N, Williams LT (1992). The fms-like tyrosine kinase, a receptor for vascular endothelial growth factor. Science 255: 989-991.

Detke MJ, Rickels M, Lucki I (1995). Active behaviors in the rat forced swimming test differentially produced by serotonergic and noradrenergic antidepressants. Psychopharmacology (Berl) 121: $66-72$.

Dew MA, Bromet EJ, Schulberg HC, Dunn LO, Parkinson DK (1987). Mental health effects of the Three Mile Island nuclear reactor restart. Am J Psychiatry 144: 1074-1077.

Duman RS (2002). Structural alterations in depression: cellular mechanisms underlying pathology and treatment of mood disorders. CNS Spectr 7: 140-142, 144-147.

Duman RS (2004). Role of neurotrophic factors in the etiology and treatment of mood disorders. Neuromolecular Med 5: 11-25.

Haidkind R, Eller M, Harro M, Kask A, Rinken A, Oreland L et al (2003). Effects of partial locus coeruleus denervation and chronic mild stress on behaviour and monoamine neurochemistry in the rat. Eur Neuropsychopharmacol 13: 19-28.

Heine VM, Zareno J, Maslam S, Joels M, Lucassen PJ (2005). Chronic stress in the adult dentate gyrus reduces cell proliferation near the vasculature and VEGF and Flk-1 protein expression. Eur J Neurosci 21: 1304-1314.

Holick KA, Lee DC, Hen R, Dulawa SC (2008). Behavioral effects of chronic fluoxetine in $\mathrm{BALB} / \mathrm{cJ}$ mice do not require adult hippocampal neurogenesis or the serotonin $1 \mathrm{~A}$ receptor. Neuropsychopharmacology 33: 406-417.

Impey S, McCorkle SR, Cha-Molstad H, Dwyer JM, Yochum GS, Boss JM et al (2004). Defining the CREB regulon: a genome-wide analysis of transcription factor regulatory regions. Cell 119: 1041-1054.

Jin K, Zhu Y, Sun Y, Mao XO, Xie L, Greenberg DA (2002). Vascular endothelial growth factor (VEGF) stimulates neurogenesis in vitro and in vivo. Proc Natl Acad Sci USA 99: 11946-11950.

Krishnan V, Nestler EJ (2008). The molecular neurobiology of depression. Nature 455: 894-902. 
Kulkarni SK, Bhutani MK, Bishnoi M (2008). Antidepressant activity of curcumin: involvement of serotonin and dopamine system. Psychopharmacology (Berl) 201: 435-442.

Lucki I (1997). The forced swimming test as a model for core and component behavioral effects of antidepressant drugs. Behav Pharmacol 8: 523-532.

MacQueen GM, Campbell S, McEwen BS, Macdonald K, Amano S, Joffe RT et al (2003). Course of illness, hippocampal function, and hippocampal volume in major depression. Proc Natl Acad Sci USA 100: 1387-1392.

Martinowich K, Manji H, Lu B (2007). New insights into BDNF function in depression and anxiety. Nat Neurosci 10: 1089-1093.

Mendez J, Kadia TM, Somayazula RK, El-Badawi KI, Cowen DS (1999). Differential coupling of serotonin 5-HT1A and 5-HT1B receptors to activation of ERK2 and inhibition of adenylyl cyclase in transfected $\mathrm{CHO}$ cells. J Neurochem 73: 162-168.

Mologni L, Sala E, Cazzaniga S, Rostagno R, Kuoni T, Puttini M et al (2006). Inhibition of RET tyrosine kinase by SU5416. J Mol Endocrinol 37: 199-212.

Muscat R, Papp M, Willner P (1992). Reversal of stress-induced anhedonia by the atypical antidepressants, fluoxetine and maprotiline. Psychopharmacology (Berl) 109: 433-438.

Newton SS, Collier EF, Hunsberger J, Adams D, Terwilliger R, Selvanayagam E et al (2003). Gene profile of electroconvulsive seizures: induction of neurotrophic and angiogenic factors. J Neurosci 23: 10841-10851.

Newton SS, Dow A, Terwilliger R, Duman R (2002). A simplified method for combined immunohistochemistry and in-situ hybridization in fresh-frozen, cryocut mouse brain sections. Brain Res Brain Res Protoc 9: 214-219.

Palmer TD, Willhoite AR, Gage FH (2000). Vascular niche for adult hippocampal neurogenesis. J Comp Neurol 425: 479-494.

Papp M, Willner P, Muscat R (1991). An animal model of anhedonia: attenuation of sucrose consumption and place preference conditioning by chronic unpredictable mild stress. Psychopharmacology (Berl) 104: 255-259.

Paxinos G, Watson C (1998). The Rat Brain in Stereotaxic Coordinates, 4th edn. Academic Press: San Diego, CA.

Quinn TP, Peters KG, De Vries C, Ferrara N, Williams LT (1993). Fetal liver kinase 1 is a receptor for vascular endothelial growth factor and is selectively expressed in vascular endothelium. Proc Natl Acad Sci USA 90: 7533-7537.

Riad M, Garcia S, Watkins KC, Jodoin N, Doucet E, Langlois X et al (2000). Somatodendritic localization of 5-HT1A and preterminal axonal localization of 5-HT1B serotonin receptors in adult rat brain. J Comp Neurol 417: 181-194.

Rogoz Z, Skuza G, Maj J, Danysz W (2002). Synergistic effect of uncompetitive NMDA receptor antagonists and antidepressant drugs in the forced swimming test in rats. Neuropharmacology 42: $1024-1030$.

Santarelli L, Saxe M, Gross C, Surget A, Battaglia F, Dulawa S et al (2003). Requirement of hippocampal neurogenesis for the behavioral effects of antidepressants. Science 301: 805-809.

Schmidt HD, Duman RS (2007). The role of neurotrophic factors in adult hippocampal neurogenesis, antidepressant treatments and animal models of depressive-like behavior. Behav Pharmacol 18: 391-418.

Segi-Nishida E, Warner-Schmidt JL, Duman RS (2008). Electroconvulsive seizure and VEGF increase the proliferation of neural stem-like cells in rat hippocampus. Proc Natl Acad Sci USA 105: 11352-11357.

Sell SL, Craft RM, Seitz PK, Stutz SJ, Cunningham KA, Thomas ML (2008). Estradiol-sertraline synergy in ovariectomized rats. Psychoneuroendocrinology 33: 1051-1060.

Sheline YI, Gado MH, Kraemer HC (2003). Untreated depression and hippocampal volume loss. Am J Psychiatry 160: 1516-1518.

Smolich BD, Yuen HA, West KA, Giles FJ, Albitar M, Cherrington JM (2001). The antiangiogenic protein kinase inhibitors SU5416 and SU6668 inhibit the SCF receptor (c-kit) in a human myeloid leukemia cell line and in acute myeloid leukemia blasts. Blood 97: 1413-1421.

Storkebaum E, Lambrechts D, Carmeliet P (2004). VEGF: once regarded as a specific angiogenic factor, now implicated in neuroprotection. Bioessays 26: 943-954.

Umberson D, Wortman CB, Kessler RC (1992). Widowhood and depression: explaining long-term gender differences in vulnerability. J Health Soc Behav 33: 10-24.

Warner-Schmidt JL, Duman RS (2007). VEGF is an essential mediator of the neurogenic and behavioral actions of antidepressants. Proc Natl Acad Sci USA 104: 4647-4652.

Warner-Schmidt JL, Duman RS (2008). VEGF as a potential target for therapeutic intervention in depression. Curr Opin Pharmacol 8: 14-19.

Weinstock M, Poltyrev T, Bejar C, Youdim MB (2002). Effect of TV3326, a novel monoamine-oxidase cholinesterase inhibitor, in rat models of anxiety and depression. Psychopharmacology (Berl) 160: 318-324.

West CH, Weiss JM (2005). A selective test for antidepressant treatments using rats bred for stress-induced reduction of motor activity in the swim test. Psychopharmacology (Berl) 182: 9-23.

Willner P (2005). Chronic mild stress (CMS) revisited: consistency and behavioural-neurobiological concordance in the effects of CMS. Neuropsychobiology 52: 90-110.

Yang SZ, Zhang LM, Huang YL, Sun FY (2003). Distribution of Flk-1 and Flt-1 receptors in neonatal and adult rat brains. Anat Rec A Discov Mol Cell Evol Biol 274: 851-856. 